\title{
Research on the Effectiveness of Different Estimation Algorithm on the Autonomous Orbit Determination of Lagrangian Navigation Constellation
}

\author{
Youtao Gao, ${ }^{1}$ Junkang Chen, ${ }^{1}$ Bo Xu, ${ }^{2}$ and Jianhua $\mathrm{Zhou}^{3}$ \\ ${ }^{1}$ College of Astronautics, Nanjing University of Aeronautics and Astronautics, Nanjing, China \\ ${ }^{2}$ College of Astronomy and Space Science, Nanjing University, Nanjing, China \\ ${ }^{3}$ Beijing Satellite Navigation Center, Beijing, China \\ Correspondence should be addressed to Youtao Gao; ytgao@nuaa.edu.cn
}

Received 7 July 2016; Revised 5 September 2016; Accepted 25 September 2016

Academic Editor: Enrico C. Lorenzini

Copyright ( 2016 Youtao Gao et al. This is an open access article distributed under the Creative Commons Attribution License, which permits unrestricted use, distribution, and reproduction in any medium, provided the original work is properly cited.

\begin{abstract}
The accuracy of autonomous orbit determination of Lagrangian navigation constellation will affect the navigation accuracy for the deep space probes. Because of the special dynamical characteristics of Lagrangian navigation satellite, the error caused by different estimation algorithm will cause totally different autonomous orbit determination accuracy. We apply the extended Kalman filter and the fading-memory filter to determinate the orbits of Lagrangian navigation satellites. The autonomous orbit determination errors are compared. The accuracy of autonomous orbit determination using fading-memory filter can improve $50 \%$ compared to the autonomous orbit determination accuracy using extended Kalman filter. We proposed an integrated Kalman fading filter to smooth the process of autonomous orbit determination and improve the accuracy of autonomous orbit determination. The square root extended Kalman filter is introduced to deal with the case of inaccurate initial error variance matrix. The simulations proved that the estimation method can affect the accuracy of autonomous orbit determination greatly.
\end{abstract}

\section{Introduction}

Deep space exploration has become a hot spot of aerospace. Several deep space probes have been launched. The autonomous navigation is important for deep space probes to deal with communication delay as well as reducing the dependency on ground stations. As early as 1968, the sextant had been used for autonomous navigation in "Apollo program" [1]. In 1999, "Deep Space 1" achieved autonomous orbit determination by tracking small celestial bodies with an optical sensor, which is the first successful on-orbit application of the deep space autonomous navigation technology [2]. The comet probe "Deep Impact" which was launched in 2005 also carried out its navigation and control automatically based on an optical navigation system with a high resolution imager [3]. In [4], Downs proposed to use X-ray pulsar radiation signal to navigate spacecraft. The rotation period of X-ray pulsar is extremely stable; therefore, time and the location of spacecraft can be determined by tracking several X-ray pulsars with given and fixed frequency [5]. The satellite navigation constellation can also provide navigation information for deep space probes. GPS can navigate the deep space probes when they are running in low-Earth orbits and medium Earth orbits. For deep space transfer orbits and deep space target orbits, the GPS is not good enough. Several researchers investigated weak GNSS signal navigation for the deep space probes [6-8]. Witternigg et al. introduced how GPS and Galileo could be used for orbit determination in future missions to the Moon [8]. Farquhar introduced a concept of using Earth-Moon libration point satellites for lunar navigation $[9,10]$. In 2005, Hill suggested placing navigation constellation on the periodic orbits in the vicinity of libration points of the Earth-Moon system to support deep space navigation [11]. Zhang and $\mathrm{Xu}$ analyzed the architecture and navigation performance of the Lagrangian point satellite navigation system [1214]. The Lagrangian navigation constellation is introduced to navigate the deep space probes autonomously. Hence, 
the navigation constellation itself should have the ability of autonomous orbit determination (AOD). The methods introduced in [1-5] can be considered as absolute navigation (or absolute autonomous orbit determination) because the estimated orbit refers to an inertial or quasi-inertial frame. Methods introduced in [6-14] can be classified as relative navigation. Relative navigation seeks optimal estimates for the position and velocity of one satellite relative to the other one.

Relative navigation usually is applied to satellites in a formation or constellation involved using GPS which restricts the spacecraft formation to near-Earth applications, such as Deep Space Mission 3 [15] and Grace project [16]. Relative navigation is primarily proposed for formation configuration control and formation reconfiguration. However, Lagrangian navigation constellation should provide absolute navigation information to deep space probes to achieve absolute navigation. Therefore, autonomous orbit determination of the Lagrangian navigation constellation is actually using the relative measurement to achieve absolute navigation. The laser interferometer space antenna (LISA) mission is an example which uses the relative range to assist the absolute orbit determination. The LISA mission which consists of three spacecraft separated by 5 million kilometers forming an equilateral triangle is a huge Michelson interferometer in space for gravitational wave detection [17]. The deep spacenetwork provides a raw estimation of the absolute positioning for the three satellites. An accurate relative positioning will be provided with a laser-based ranging measurement in order to obtain an accuracy of positioning of tens of meters [18, 19]. Psiaki [20] and Markley [21] suggested using crosslink range, attitude information, and an optical tracker to determine the orbits autonomously. Yim et al. proposed using optical tracking and attitude information to find the direction vector between the two spacecraft and determine both orbits [22]. But these methods require extensive hardware development. In order to reduce the operational cost, size, and weight of spacecraft for formation missions, crosslink range can be used as the only measurement for orbit determination of a constellation. However, for the Earth navigation satellite constellation, there is a rank deficiency problem when only crosslink range is used to determine the orbit [23, 24]. Hill's study illustrated that the rank deficiency problem does not exist for the Lagrangian navigation satellites because of the special dynamics near the libration points [11]. Thus, the Lagrangian navigation satellites can autonomously determine their orbits using only crosslink range. In [11], Hill discussed the unique distribution of Lagrangian orbit from the view of dynamics, which theoretically proved the autonomy of the Lagrangian navigation constellation. From the perspective of identifiability of epoch state, Qian et al. verified the feasibility of AOD for satellites in quasiperiodic orbits about the EarthMoon libration point [25]. Based on circular restricted threebody problem (CR3BP), Du et al. researched the autonomous orbit determination method of satellites in halo orbits, and only crosslink range was used as observation [26]. In [23], Gao et al. discussed the feasibility of autonomous orbit determination using only the crosslink range measurement for a combined Lagrangian navigation constellation and GNSS.

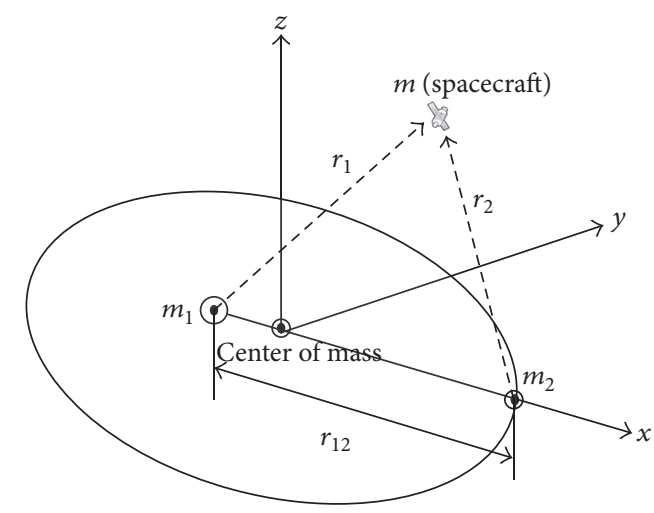

FIGURE 1: Diagram of the circular restricted three-body problem in $o-x y z$ frame.

The most widely used algorithm for Lagrangian navigation satellite is extended Kalman filter (EKF) method. However, EKF is based on linearization of the system dynamics and the assumption of Gaussian process/measurement noise. These can seriously affect the performance of the state estimation and even lead to divergence. Unscented Kalman filter (UKF) which is based on the unscented transform can achieve higher accuracy than EKF while the added computational cost is not significant. More importantly, UKF is robust with respect to the initial conditions. Therefore, Sun et al. introduced UKF to relative navigation for multiple spacecraft formation flying [27]. Giannitrapani et al. analyze the performance of EKF and UKF for the localization of a spacecraft [28]. In order to improve the robustness and stability accuracy, Wang and $\mathrm{Gu}$ applied fault tolerant UKF in autonomous determination of relative orbit for satellite formation flying [29]. Rigatos introduced the technical analysis and implementation cost assessment of sigma-point Kalman filtering and particle filtering in autonomous navigation systems [30]. Reali and Palmerini provided a preliminary comparison of different estimation techniques to be used in formation flying navigation [31].

Since the CR3BP is sensitive to the state error and calculation error, the AOD of Lagrangian navigation satellite may refer to the accuracy of the estimation algorithms. One factor which must be considered in the AOD of Lagrangian navigation constellation is to prevent the divergence of $\mathrm{AOD}$ error. Therefore, we introduce four estimation methods to achieve the AOD of Lagrangian navigation constellation to analyze the effect on AOD by estimation method.

\section{Dynamical Model of Lagrangian Navigation Satellite}

For satellites in Lagrangian point orbits, the equation of CR3BP should be an appropriate model to describe the satellites' dynamical characteristics. Consider two massive bodies $m_{1}$ and $m_{2}$ moving under the action of just their mutual gravitation, and let their orbit around each other be a circle of radius $r_{12}$. As shown in Figure 1, a noninertial, comoving frame of reference $o-x y z$ is defined. The origin 
of frame $o-x y z$ lies at the center of mass of the two-body system. The positive $x$ direction goes from $m_{1}$ to $m_{2}$. The positive $y$-axis is parallel to the velocity vector. The $z$-axis is perpendicular to the orbital plane. Now the third body of mass $m$ which is vanishingly small compared to the primary masses $m_{1}$ and $m_{2}$ is introduced. We assume that the mass $m$ is so small that it has no effect on the motion of the primary bodies. This is called the restricted three-body problem.

The nondimensional equations of motion for the CR3BP are shown as follows [32]:

$$
\begin{aligned}
\ddot{x}-2 \dot{y} & =x-(1-\mu) \frac{x+\mu}{r_{1}^{3}}-\mu \frac{x+\mu-1}{r_{2}^{3}}, \\
\ddot{y}+2 \dot{x} & =\left(1-\frac{1-\mu}{r_{1}^{3}}-\frac{\mu}{r_{2}^{3}}\right) y, \\
\ddot{z} & =\left(\frac{\mu-1}{r_{1}^{3}}-\frac{\mu}{r_{2}^{3}}\right) z,
\end{aligned}
$$

where $m_{1}$ and $m_{2}$ are the masses of the two primaries. $r_{1}$ and $r_{2}$ are the distances of the spacecraft from the massive and secondary primaries, respectively. $r_{1}, r_{2}$, and $\mu$ are given by

$$
\begin{aligned}
& r_{1}=\sqrt{(x+\mu)^{2}+y^{2}+z^{2}}, \\
& r_{2}=\sqrt{(x+\mu-1)^{2}+y^{2}+z^{2}}, \\
& \mu=\frac{m_{2}}{m_{1}+m_{2}} .
\end{aligned}
$$

where $r_{12}$ is the distance between the two primaries. $G$ is the Universal Constant of Gravitation.

Equations (1) have five equilibrium points which are called Lagrange points (or libration point), as shown in Figure 2.

Three Lagrange points on the $x$-axis are unstable, and two Lagrange points which form an equilateral triangle with the two primary bodies in the $x-y$ plane are stable. Many interesting periodic orbits exist in the vicinity of the Lagrange points no matter whether they are stable. The Lagrange navigation satellite constellation discussed in this paper is distributed in these periodic orbits.

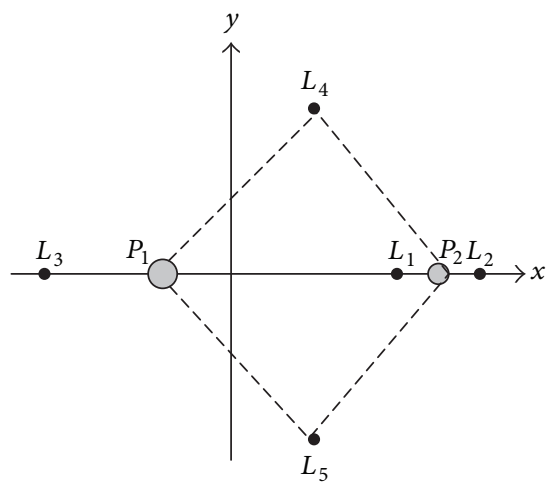

FIGURE 2: Schematic location of the five Lagrange points in the CR3BP.

\section{AOD of Lagrangian Navigation Constellation with Different Estimation Methods}

EKF method is a commonly used method for AOD of satellite. EKF is summarized as follows [33]:

$$
\begin{aligned}
\widehat{X}_{k} & =\Phi_{k, k-1} \widehat{X}_{k-1}+K_{k}\left(\rho_{k}-H_{k} \Phi_{k, k-1} \widehat{X}_{k-1}\right), \\
K_{k} & =P_{k} H_{k}^{T} R_{k}^{-1}, \\
P_{k} & =\left(I-K_{k} H_{k}\right) P_{k, k-1}, \\
P_{k, k-1} & =\Phi_{k, k-1} P_{k-1} \Phi_{k, k-1}^{T}+\Gamma_{k, k-1} Q_{k-1} \Gamma_{k, k-1}^{T},
\end{aligned}
$$

where $\widehat{X}_{k-1}$ is the estimated state at $t_{k-1} . \Phi\left(t_{k}, t_{k-1}\right)$ is the transition matrix. $P_{k}$ is the covariance matrix. $H_{k}$ is the mapping matrix that relates the observation deviation vector to the state deviation vector. $K_{k}$ is the Kalman gain matrix. In this paper, only crosslink range between two satellites is used as observation. The crosslink range is described as

$$
\rho_{i, j}=\sqrt{\left(x_{i}-x_{j}\right)^{2}+\left(y_{i}-y_{j}\right)^{2}+\left(z_{i}-z_{j}\right)^{2}} \text {. }
$$

So

$$
H_{k}=\left.\frac{\partial \rho\left(\mathbf{x}_{k}, k\right)}{\partial \mathbf{x}_{k}^{T}}\right|_{\mathbf{x}_{k, k-1}}
$$

Two periodic orbits around $L_{1}$ and $L_{2}$, respectively, are chosen to achieve the simulation. The initial states of the two orbits are shown in Table 1 . The values are nondimensional. $10 \mathrm{~m}$ initial state errors and $10 \mathrm{~m}$ measurement noise are added. In Figures 3 and 4, the orbits based on different initial state is shown. As we can see, the trajectory will diverge within a short time if the initial state error is $10 \mathrm{~m}$. Therefore, the initial state error and the measurement noise are chosen based on the navigation requirement, not the ability of the existing technology. 
TABLE 1: Initial states of the periodic orbits around Lagrange points $L_{1}$ and $L_{2}$.

\begin{tabular}{lcc}
\hline Satellites & $L_{1}$ & $L_{2}$ \\
\hline$x(L)$ & 0.8440212401521470 & 1.1726789595745000 \\
$y(L)$ & 0.0000000000000000 & 0.0000000000000000 \\
$z(L)$ & 0.0592695845762629 & 0.0834298981288340 \\
$\dot{x}(L / T)$ & 0.00000000000000000 & 0.0000000000000000 \\
$\dot{y}(L / T)$ & -0.0053009560909076 & -0.1864489128036080 \\
$\dot{z}(L / T)$ & 0.0000000000000000 & 0.0000000000000000 \\
\hline
\end{tabular}

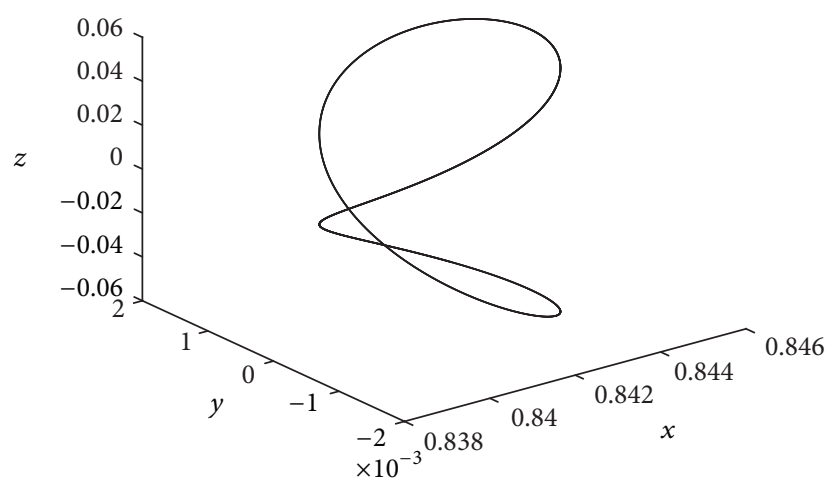

FIgURE 3: The periodic orbit based on accurate initial state.

The initial covariance matrix is defined as

$$
P\left(t_{0}\right)=\left[\begin{array}{cccccc}
\sigma_{x_{0}}^{2} & 0 & 0 & 0 & 0 & 0 \\
0 & \sigma_{y_{0}}^{2} & 0 & 0 & 0 & 0 \\
0 & 0 & \sigma_{z_{0}}^{2} & 0 & 0 & 0 \\
0 & 0 & 0 & \sigma_{\dot{x}_{0}}^{2} & 0 & 0 \\
0 & 0 & 0 & 0 & \sigma_{\dot{y}_{0}}^{2} & 0 \\
0 & 0 & 0 & 0 & 0 & \sigma_{\dot{z}_{0}}^{2}
\end{array}\right],
$$

where $\sigma_{x_{0}}, \sigma_{y_{0}}$, and $\sigma_{z_{0}}$ are the initial position error in three coordinate axes and $\sigma_{\dot{x}_{0}}, \sigma_{\dot{y}_{0}}$, and $\sigma_{\dot{z}_{0}}$ are the initial velocity error in three coordinate axes.

Figures 5 and 6 show the results of AOD of Lagrangian navigation satellite using EKF. As we can see, the maximum errors in three axes of $L_{1}$ are $541 \mathrm{~m}, 254 \mathrm{~m}$ and $168 \mathrm{~m}$. The maximum errors in three axes of $L_{2}$ are $555 \mathrm{~m}, 195 \mathrm{~m}$, and $263 \mathrm{~m}$. And the estimation process is divergent.

Since CR3BP is sensitive to calculation error, the state estimation error covariance matrix of EKF may lose its properties of positive definite when the computations are carried out with finite digit arithmetic of the computer. Nonpositive definite covariance matrix will cause the gain matrix to lose its function gradually and lead to divergence of AOD error. In order to suppress the divergence of estimation process, the fading-memory filter (FMF) is introduced to improve the AOD characteristics. The FMF is essentially the old estimate plus a gain times a residual (difference between current measurement and previous estimate). The difference between the EKF and FMF is that the FMF is recursive

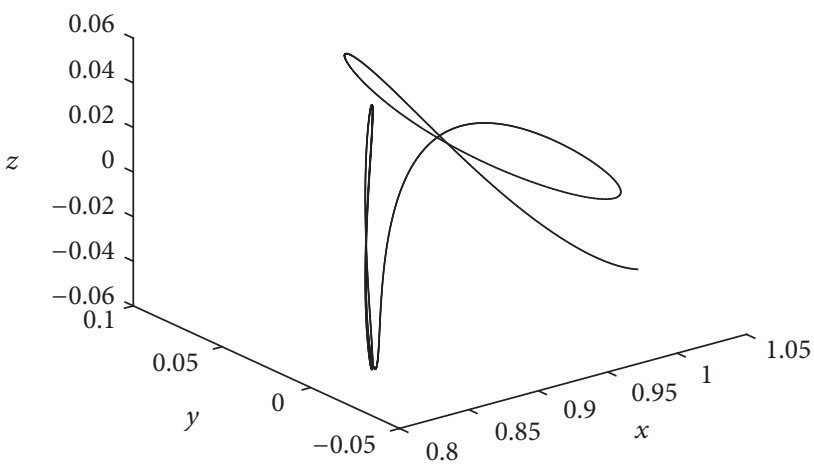

FIGURE 4: The orbit based on the initial state with $10 \mathrm{~m}$ error.
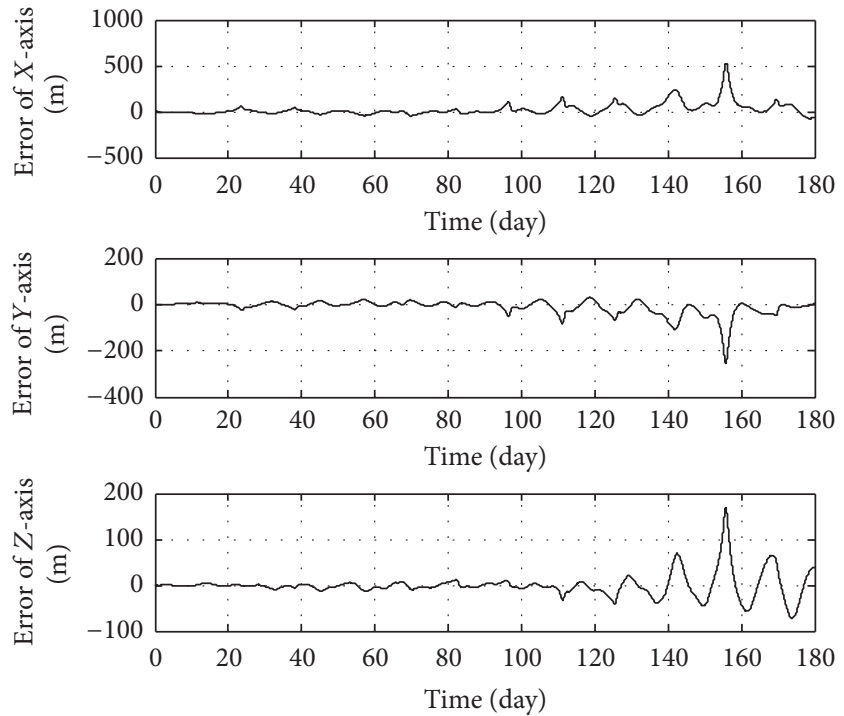

FIgURE 5: The AOD position error of $L_{1}$ using EKF.
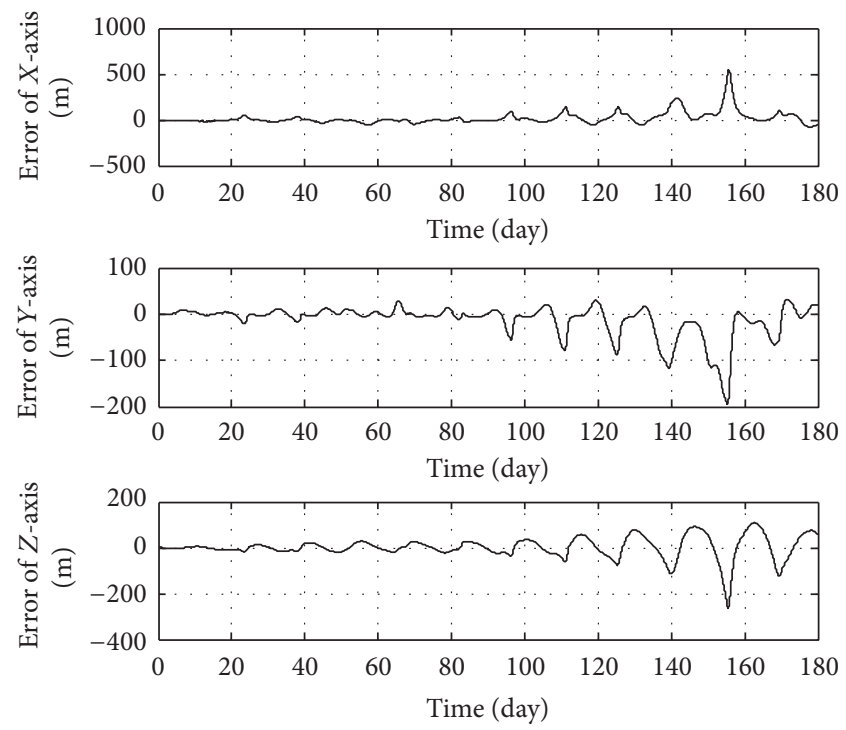

FIGURE 6: The AOD position error of $L_{2}$ using EKF. 

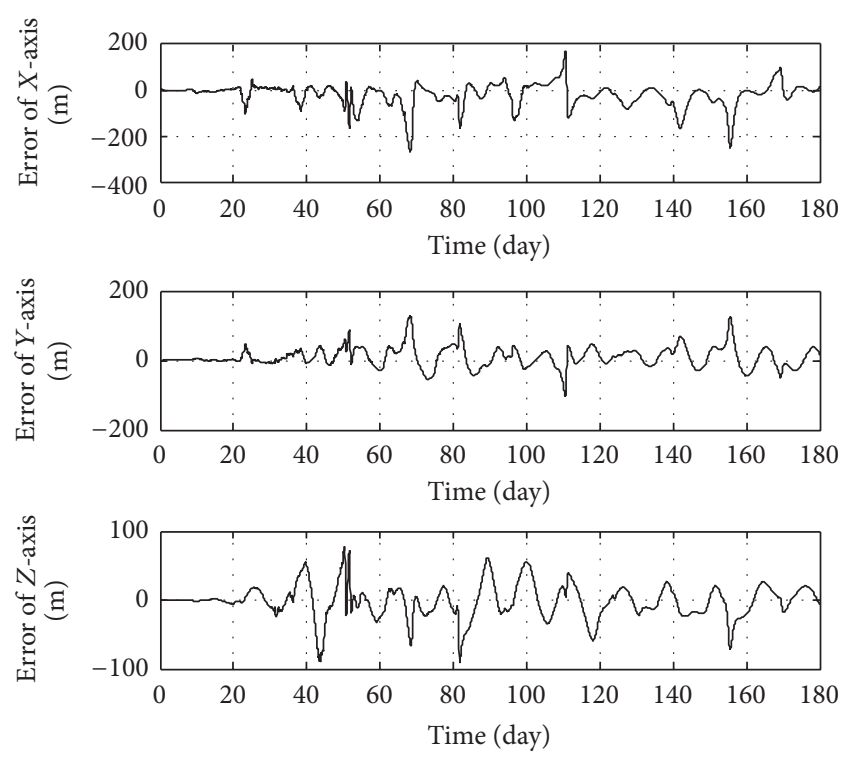

FIgURE 7: The AOD position error of $L_{1}$ using FMF.
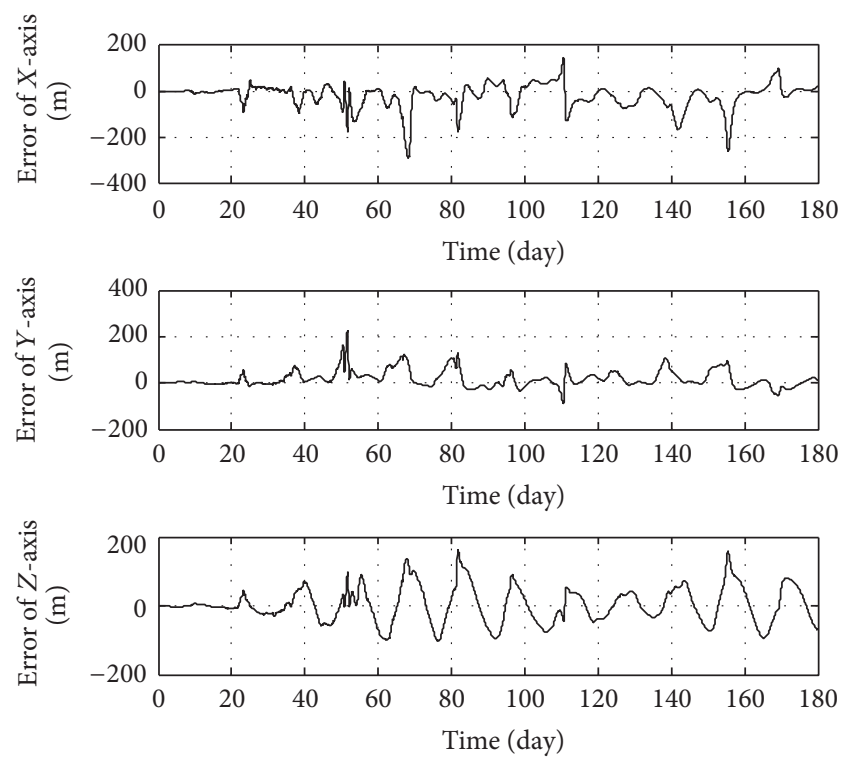

FIgure 8: The AOD position error of $L_{2}$ using FMF.

and weights new measurements more heavily than older measurements [34].

The basic equations of FMF are listed as follows [33]:

$$
\begin{aligned}
\widehat{X}_{k} & =\Phi_{k, k-1} \widehat{X}_{k-1}+K_{k}\left[\rho_{k}-H_{k} \Phi_{k, k-1} \widehat{X}_{k-1}\right], \\
K_{k} & =P_{k} H_{k}^{T} R_{k}^{-1}, \\
P_{k} & =\left(I-K_{k} H_{k}\right) P_{k, k-1}, \\
P_{k, k-1} & =\Phi_{k, k-1} P_{k-1} \Phi_{k, k-1}^{T} e^{C_{k-1}}+\Gamma_{k, k-1} Q_{k-1} \Gamma_{k, k-1}^{T} .
\end{aligned}
$$

FMF raises the role of new observation data and reduces the influence of old observation to the filter in order to suppress the filtering divergence. Figures 7 and 8 show the AOD position errors of $L_{1}$ and $L_{2}$ using FMF. It can be seen that the divergence of errors is suppressed by using FMF method. The accuracy of the AOD is improved as well.

One disadvantage of FMF is that the oscillation amplitude of filtering error at early stage is larger. Comparing Figure 5 with Figure 7, we can see that estimation process of EKF is relatively ideal during the first $100 \mathrm{~s}$. Therefore, we can use EKF to determine the orbit of Lagrangian navigation satellite at the early stage of the AOD. When the AOD error is bigger than a threshold value, the estimation method will be switching to FMF. We call this method integrated Kalman fading filter (IKFF) which is illustrated as

$$
\begin{aligned}
& \widehat{X}_{k}=\Phi_{k, k-1} \widehat{X}_{k-1}+K_{k}\left[\rho_{k}-H_{k} \Phi_{k, k-1} \widehat{X}_{k-1}\right], \\
& K_{k}=P_{k} H_{k}^{T} R_{k}^{-1}, \\
& P_{k}=\left(I-K_{k} H_{k}\right) P_{k, k-1}, \\
& P_{k, k-1} \\
& = \begin{cases}\Phi_{k, k-1} P_{k-1} \Phi_{k, k-1}^{T}+\Gamma_{k, k-1} Q_{k-1} \Gamma_{k, k-1}^{T}, & \text { if } e \geq e_{s}, \\
\Phi_{k, k-1} P_{k-1} \Phi_{k, k-1}^{T} e^{C_{k-1}}+\Gamma_{k, k-1} Q_{k-1} \Gamma_{k, k-1}^{T}, & \text { if } e<e_{s},\end{cases}
\end{aligned}
$$

where $e_{s}$ is the threshold value of the AOD error. Since the estimation error cannot be got in practical applications, the residual can be used to approximate the threshold value of estimation error. The linearized relationship between the residual and the $\mathrm{AOD}$ error can be expressed as

$$
y=C e,
$$

where $y$ is the residual and $C$ is denoted as

$$
C=\left[\begin{array}{llllll}
\frac{\hat{x}-\hat{x}_{2}}{\rho} & \frac{\hat{y}-\hat{y}_{2}}{\rho} & \frac{\hat{z}-\widehat{z}_{2}}{\rho} & 0 & 0 & 0
\end{array}\right] .
$$

$\left[\begin{array}{lll}\hat{x} & \hat{y} & \widehat{z}\end{array}\right]^{T}$ and $\left[\begin{array}{lll}\hat{x}_{2} & \widehat{y}_{2} & \widehat{z}_{2}\end{array}\right]^{T}$ are the estimated position variable of Lagrangian satellite 1 and Lagrangian satellite 2, respectively. $\rho$ is the crosslink range between these two satellites.

The AOD results of IKFF are shown in Figures 9 and 10. We list the maximum error of the above three methods in Tables 2 and 3. As shown in Table 2, the maximum error of $L_{1}$ satellite in three axes using IKFF reduced to $204 \mathrm{~m}, 104 \mathrm{~m}$, and $62 \mathrm{~m}$. Meanwhile, oscillation characteristic at the early stage of AOD is improved in Figure 9. The similar improvement of AOD can be seen about $L_{2}$ satellite. Therefore, as long as the threshold value is selected appropriately, IKFF will produce better AOD results than FMF or EKF.

To overcome the divergence of AOD error caused by the state estimation error covariance matrix losing its properties of positive definite symmetric, we also introduce the square root extended Kalman filter (SR-EKF) to estimate the state of 

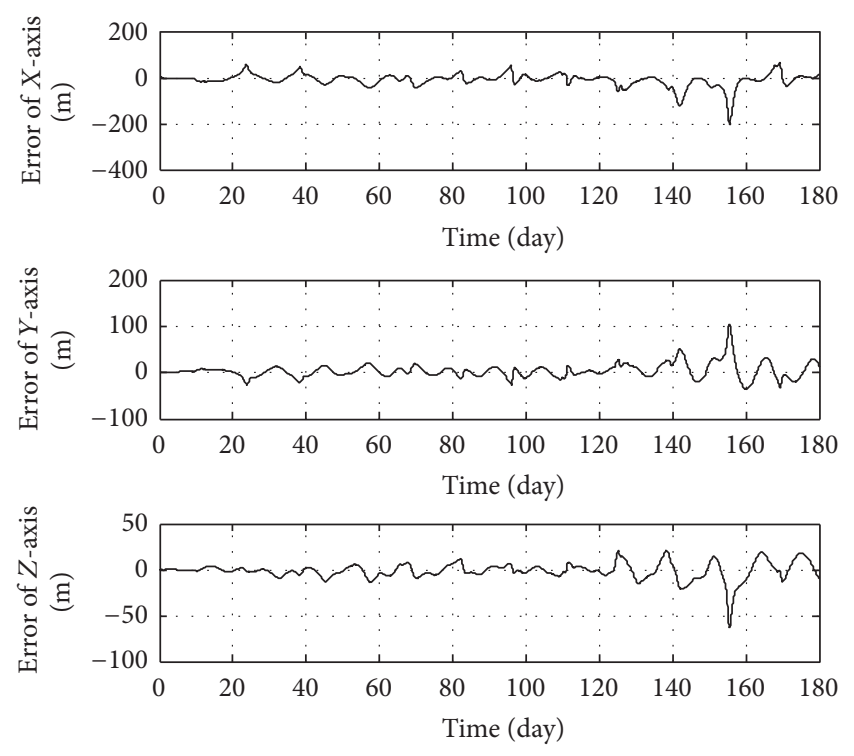

FIGURE 9: The AOD position error of $L_{1}$ using IKFF.
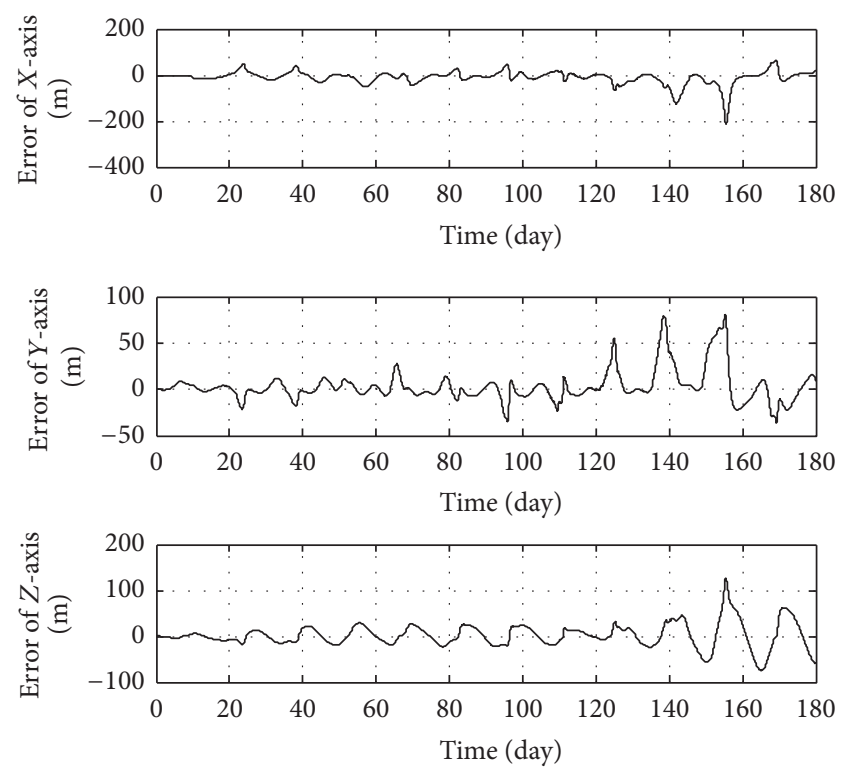

FIgURE 10: The AOD position error of $L_{2}$ using IKFF.

the Lagrangian satellite using only crosslink range. The SREKF is described as follows [33]:

$$
\begin{aligned}
\widehat{X}_{k} & =\Phi_{k, k-1} \widehat{X}_{k-1}+K_{k}\left[\rho_{k}-H_{k} \Phi_{k, k-1} \widehat{X}_{k-1}\right], \\
\widehat{X}_{0} & =E\left[X_{0}\right], \\
K_{k} & =a_{k} S_{k, k-1} F_{k}, \\
& \quad F_{k}=S_{k, k-1}^{T} H_{k}^{T}, a_{k}=\left[F_{k}^{T} F_{k}+R_{k}\right]^{-1}, \\
S_{k, k-1} & =\Phi_{k, k-1} S_{k-1},
\end{aligned}
$$

TABLE 2: The maximum AOD error of $L_{1}$ using different methods.

\begin{tabular}{lccc}
\hline & $\begin{array}{c}\text { Maximum error } \\
\text { in } x \text {-axis/m }\end{array}$ & $\begin{array}{c}\text { Maximum error } \\
\text { in } y \text {-axis/m }\end{array}$ & $\begin{array}{c}\text { Maximum error } \\
\text { in } z \text {-axis } / \mathrm{m}\end{array}$ \\
\hline EKF & 541 & 253 & 168 \\
FMF & 273 & 129 & 92 \\
IKFF & 204 & 104 & 62 \\
\hline
\end{tabular}

TABLE 3: The maximum AOD error of $L_{2}$ using different methods.

\begin{tabular}{lccc}
\hline & $\begin{array}{c}\text { Maximum error } \\
\text { in } x \text {-axis/m }\end{array}$ & $\begin{array}{c}\text { Maximum error } \\
\text { in } y \text {-axis } / \mathrm{m}\end{array}$ & $\begin{array}{c}\text { Maximum error } \\
\text { in } z \text {-axis } / \mathrm{m}\end{array}$ \\
\hline EKF & 554 & 195 & 262 \\
FMF & 296 & 226 & 163 \\
IKFF & 215 & 81 & 128 \\
\hline
\end{tabular}

TABLE 4: The maximum AOD error of $L_{1}$ using SR-EKF with different $P_{0}$.

\begin{tabular}{lccc}
\hline & $\begin{array}{c}\text { Maximum error } \\
\text { in } x \text {-axis/m }\end{array}$ & $\begin{array}{c}\text { Maximum error } \\
\text { in } y \text {-axis/m }\end{array}$ & $\begin{array}{c}\text { Maximum error } \\
\text { in } z \text {-axis } / \mathrm{m}\end{array}$ \\
\hline$P_{0}=10^{-16}$ & 17936 & 8507 & 4378 \\
$P_{0}=10^{-14}$ & 853 & 393 & 184 \\
$P_{0}=10^{-12}$ & 404 & 186 & 88 \\
$P_{0}=10^{-10}$ & 377 & 178 & 104 \\
\hline
\end{tabular}

TABLE 5: The maximum AOD error of $L_{2}$ using SR-EKF with different $P_{0}$.

\begin{tabular}{lccc}
\hline & $\begin{array}{c}\text { Maximum error } \\
\text { in } x \text {-axis/m }\end{array}$ & $\begin{array}{c}\text { Maximum error } \\
\text { in } y \text {-axis/m }\end{array}$ & $\begin{array}{c}\text { Maximum error } \\
\text { in } z \text {-axis } / \mathrm{m}\end{array}$ \\
\hline$P_{0}=10^{-16}$ & 17290 & 5216 & 6532 \\
$P_{0}=10^{-14}$ & 790 & 283 & 301 \\
$P_{0}=10^{-12}$ & 374 & 134 & 154 \\
$P_{0}=10^{-10}$ & 365 & 107 & 134 \\
\hline
\end{tabular}

$$
\begin{aligned}
& P_{0}=S_{0} S_{0}^{T}, \\
& S_{k}=S_{k, k-1}\left[I-a_{k} r_{k} F_{k} F_{k}^{T}\right], \quad r_{k}=\frac{1}{1 \pm \sqrt{a_{k} R_{k}}} .
\end{aligned}
$$

The state estimation error covariance matrix $P$ is replaced by $S S^{T}$ where $S$ is the square root of $P$ [35]. Here we define the order of $\sigma_{x_{0}}^{2}, \sigma_{y_{0}}^{2}$, and $\sigma_{z_{0}}^{2}$ as $P_{0}$. This method can guarantee the $P$ is symmetric nonnegative definite matrix at any time. In Tables 4 and 5, we give the maximum error of AOD of $L_{1}$ satellite and $L_{2}$ satellite with different initial state estimation error covariance matrix. As we can see, with a small initial value of error variance matrix, SR-EKF algorithm cannot estimate the state very well. The estimation error using SREKF is bigger than the EKF results. However, the accuracy of AOD is improved with the increase of initial error covariance matrix. Thus, the effectiveness of SR-EKF method is affected by the initial value of state estimation error covariance matrix $P$. SR-EKF is useful when the initial error variance matrix is not accurate. However, the AOD error will increase when the 

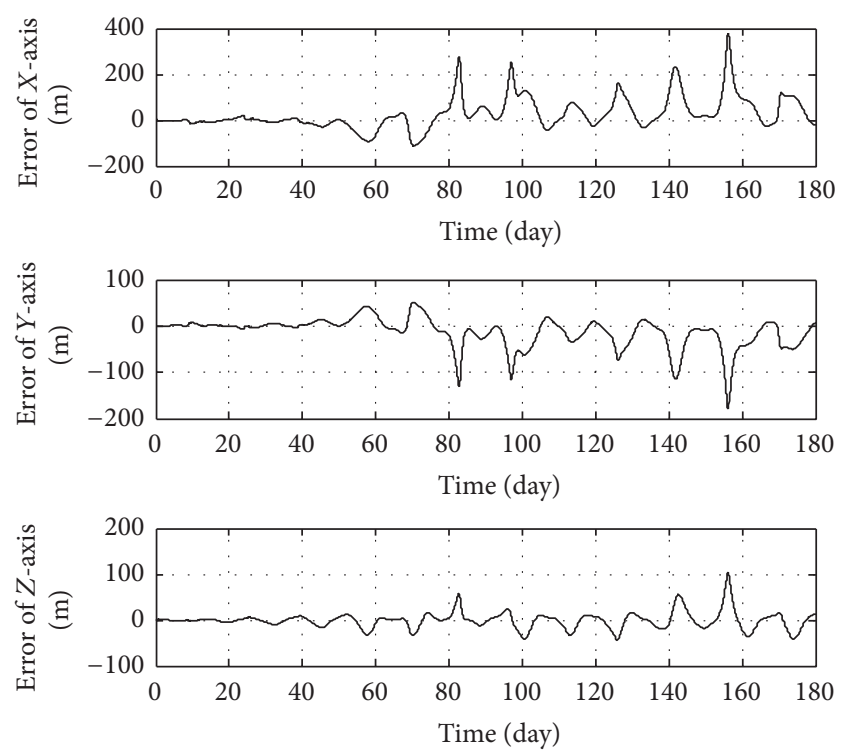

FIgURE 11: The AOD position error of $L_{1}$ using SR-EKF with $P_{0}=$ $10^{-10}$.
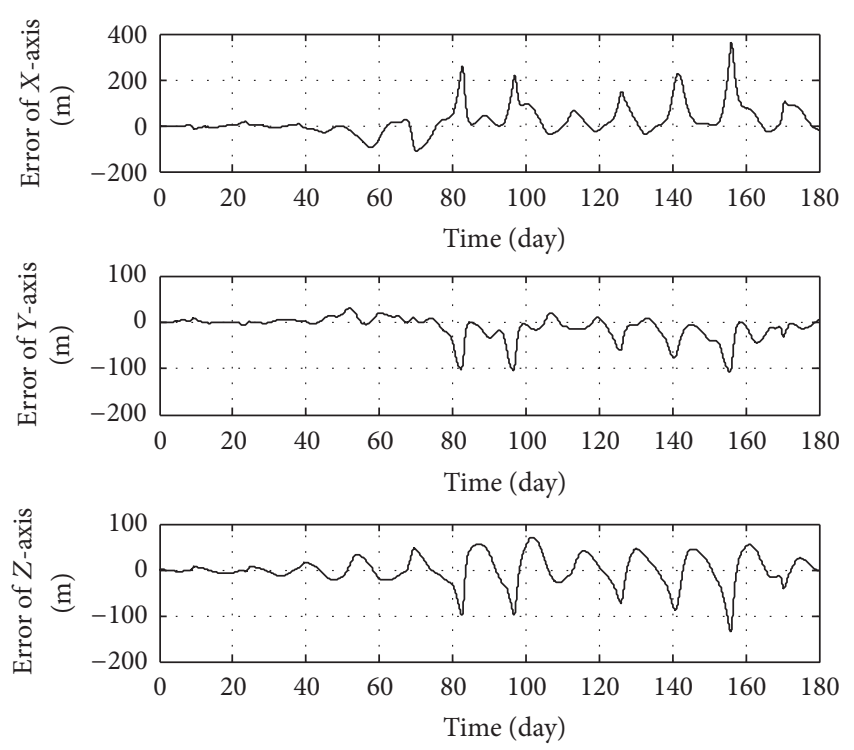

FIgURE 12: The AOD position error of $L_{2}$ using SR-EKF with $P_{0}=$ $10^{-10}$.

initial error variance matrix is bigger than a boundary. In our simulation, the best AOD result coincides with the $P_{0}=10^{-10}$. Figures 11 and 12 exhibit the AOD error using SR-EKF under the same initial condition as the above three methods with $P_{0}=10^{-10}$. Even when the initial error variance matrix is not accurate, the precision of AOD of two Lagrangian satellites is better than EKF.

\section{Conclusion}

Since the special dynamical characteristics of CR3BP, the AOD accuracy of the Lagrangian navigation constellation is sensitive to all the errors introduced by the AOD process.
Different estimation methods will cause great different AOD accuracy.

Four estimation methods are used to determinate the orbit of Lagrangian navigation constellation. EKF is a commonly used method, but it is not the best choice for the AOD of Lagrangian navigation constellation. The accuracy of AOD using FMF can improve 50\% more than using EKF. And the divergence of the AOD error is suppressed.

We proposed a new method, IKFF, to estimate the state of Lagrangian navigation satellite. The accuracy of AOD using IKFF can improve 20\% more than using FMF. Furthermore, IKFF can smooth the process of the AOD.

For the case of not accurate initial error variance matrix, SR-EKF can be chosen as the AOD estimation method. The accuracy of SR-EKF is close to the FMF.

In order to improve the AOD accuracy, all the factors which will cause errors should be analyzed. As the estimation methods, we will find other advanced technologies to get a much more accurate AOD of Lagrangian navigation satellite.

\section{Competing Interests}

The authors declare that they have no competing interests.

\section{Acknowledgments}

This study was supported by the basic scientific research fund of national defense (no. 2016110C019), the Natural Science Foundation of Jiangsu Province (no. BK20160811), Shanghai Deep Space Detection Technology Key Laboratory Open Funding (no. DS2016-01), and the specialized research fund for the doctoral program of higher education of china (no. 20133218120037).

\section{References}

[1] R. H. Battin, An Introduction to the Mathematics and Methods of Astrodynamics, AIAA Education Series, American Institute of Aeronautics and Astronautics (AIAA), Reston, Va, USA, 1999.

[2] S. Bhaskaran, J. E. Riedel, S. P. Synnott, and T. C. Wang, “The deep space 1 autonomous navigation system: a post-flight analysis," in Proceedings of the AIAA/AAS Astrodynamics Specialist Conference, AIAA-2000-3935, pp. 42-52, Denver, Colo, USA, August 2000.

[3] N. Mastrodemos, D. G. Kubitschek, and S. P. Synnott, "Autonomous navigation for the Deep Impact mission encounter with comet Tempel 1," Space Science Reviews, vol. 117, no. 1-2, pp. 95-121, 2005.

[4] G. S. Downs, "Interplanetary navigation using pulsating radio sources," NASA Technical Reports N74-34150/4, 1974.

[5] S. I. Sheikh, D. J. Pines, P. S. Ray, K. S. Wood, M. N. Lovellette, and M. T. Wolff, "Spacecraft navigation using X-ray pulsars," Journal of Guidance, Control, and Dynamics, vol. 29, no. 1, pp. 49-63, 2006.

[6] P. F. Silva, H. D. Lopes, T. R. Peres et al., "Weak GNSS signal navigation to the moon," in Proceedings of the 26th International Technical Meeting of the Satellite Division of the Institute of Navigation (ION GNSS '13), pp. 3357-3367, Nashville, Tenn, USA, September 2013. 
[7] M. Manzano-Jurado, J. Alegre-Rubio, A. Pellacani et al., "Use of weak GNSS signals in a mission to the moon," in Proceedings of the 7th ESA Workshop on Satellite Navigation Technologies and European Workshop on GNSS Signals and Signal Processing (NAVITEC '14), 2014.

[8] N. Witternigg, G. Obertaxer, M. Schönhuber et al., "Weak GNSS signal navigation for Lunar exploration missions," in Proceedings of the 28th International Technical Meeting of The Satellite Division of the Institute of Navigation (ION GNSS '15), pp. 3928-3944, Tampa, Fla, USA, September 2015.

[9] R. W. Farquhar, "Lunar communications with libration-point satellites," Journal of Spacecraft and Rockets, vol. 4, no. 10, pp. 1383-1384, 1967.

[10] R. W. Farquhar, "The control and use of libration point satellites," NASA Technical Report TR R-346, 1970.

[11] K. A. Hill, Autonomous navigation in libration point orbits [Ph.D. thesis], University of Colorado, Boulder, Colo, USA, 2007.

[12] L. Zhang and B. Xu, "A universe light house-candidate architectures of the libration point satellite navigation system," Journal of Navigation, vol. 67, no. 5, pp. 737-752, 2014.

[13] L. Zhang and B. Xu, "Navigation performance of the libration point satellite navigation system in cislunar space," Journal of Navigation, vol. 68, no. 2, pp. 367-382, 2015.

[14] L. Zhang and B. Xu, "Navigation performance of the libration point satellite navigation system for future Mars exploration," Journal of Navigation, vol. 69, no. 1, pp. 41-56, 2016.

[15] K. Lau, S. Lichten, L. Young, and B. Haines, "An innovative deep space application of GPS technology for formation flying spacecraft," in Proceedings of the Guidance, Navigation, and Control Conference, American Institute of Aeronautics and Astronautics, San Diego, Calif, USA, July 1996.

[16] Y. Long, D. Xurong, and X. Dongmei, "Precise navigation for GRACE formation flying," in Proceedings of the 1st International Symposium on Systems and Control in Aerospace and Astronautics, pp. 792-797, Harbin, China, January 2006.

[17] The LISA Study Team, Laser Interferometer Space Antenna for the Detection and Observation of Gravitational Waves: Pre-Phase A Report, Max-Planck-Institute for Quantum Optics, 1998.

[18] J. J. Esteban Delgado, A. F. Garcia Marín, I. Bykov, G. Heinzel, and K. Danzmann, "Free-space laser ranging and data communication," in Proceedings of the 6th Workshop on Positioning, Navigation and Communication (WPNC '09), pp. 275-281, IEEE, Hannover, Germany, March 2009.

[19] J. J. Esteban, A. F. García, J. Eichholz et al., "Optical ranging and data communication in space-based applications," in Proceedings of the 7th Workshop on Positioning, Navigation and Communication (WPNC '10), pp. 19-22, Dresden, Germany, March 2010.

[20] M. L. Psiaki, "Autonomous orbit determination for two spacecraft from relative position measurements," Journal of Guidance, Control, and Dynamics, vol. 22, no. 2, pp. 305-312, 1999.

[21] F. L. Markley, "Autonomous navigation using landmark and intersatellite data," in Proceedings of the AIAA/AAS Astrodynamics Conference, AIAA Paper 1984-1987, Seattle, Wash, USA, 1984.

[22] J. R. Yim, J. L. Crassidis, and J. L. Junkins, "Autonomous orbit navigation of two spacecraft system using relative line of sight measurements," in Proceedings of the AAS/AIAA Astrodynamics Specialist Conference, AAS Paper 04-257, Maui, Hawaii, USA, 2004.
[23] Y. T. Gao, B. Xu, and L. Zhang, "Feasibility study of autonomous orbit determination using only the crosslink range measurement for a combined navigation constellation," Chinese Journal of Aeronautics, vol. 27, no. 5, pp. 1199-1210, 2014.

[24] K. Hill and G. H. Born, "Autonomous interplanetary orbit determination using satellite-to-satellite tracking," Journal of Guidance, Control, and Dynamics, vol. 30, no. 3, pp. 679-686, 2007.

[25] Y.-J. Qian, W.-X. Jing, C.-S. Gao, and W.-S. Wei, "Autonomous orbit determination for quasi-periodic orbit about the translunar libration point," Journal of Astronautics, vol. 34, no. 5, pp. 625-633, 2013.

[26] L. Du, Z. Zhang, L. Yu, and S. Chen, "SST orbit determination of halo-LMO constellation in CRTBP," Cehui Xuebao/Acta Geodaetica et Cartographica Sinica, vol. 42, no. 2, pp. 184-190, 2013.

[27] D. Sun, F. Q. Zhou, and J. Zhou, "Relative navigation based on UKF for multiple spacecraft formation flying," in Proceedings of the AIAA Guidance, Navigation, and Control Conference and Exhibit, Providence, RI, USA, August 2004.

[28] A. Giannitrapani, N. Ceccarelli, F. Scortecci, and A. Garulli, "Comparison of EKF and UKF for spacecraft localization via angle measurements," IEEE Transactions on Aerospace and Electronic Systems, vol. 47, no. 1, pp. 75-84, 2011.

[29] H. Wang and X. M. Gu, "Autonomous determination of relative orbit for satellite formation flying using fault tolerant UKF," in Proceedings of the IEEE International Conference on Mechatronics and Automation (ICMA '09), pp. 3905-3909, Changchun, China, August 2009.

[30] G. G. Rigatos, “Technical analysis and implementation cost assessment of sigma-point Kalman filtering and particle filtering in autonomous navigation systems," in Proceedings of the 2010 IEEE 71st Vehicular Technology Conference (VTC '10Spring), IEEE, Taipei, Taiwan, May 2010.

[31] F. Reali and G. Palmerini, "Estimate problems for satellite clusters," in Proceedings of the IEEE Aerospace Conference (AC '08), Big Sky, Mont, USA, March 2008.

[32] L. Liu and X. Y. Hou, Deep Space Probe Orbit Mechanics, Publishing House of Electronics Industry, Beijing, China, 2012.

[33] M. Y. Fu, Z. H. Deng, and L. P. Yan, Kalman Filtering Theory and Application in the Navigation System, Science Press, Beijing, China, 2010.

[34] P. Zarchan and H. Musoff, Fundamentals of Kalman Filtering: A Practical Approach, Progress in Astronautics and Aeronautics, American Institute of Aeronautics and Astronautics(AIAA), 4th edition, 2015.

[35] R. Van Der Merwe and E. A. Wan, "The square-root unscented Kalman filter for state and parameter-estimation," in Proceedings of the International Conference on Acoustics, Speech, and Signal Processing, Salt Lake City, Utah, USA, May 2001. 


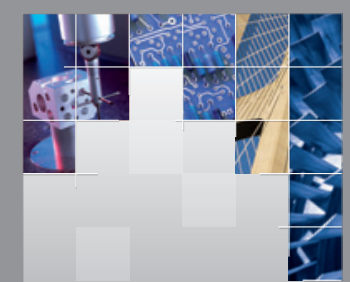

\section{Enfincering}
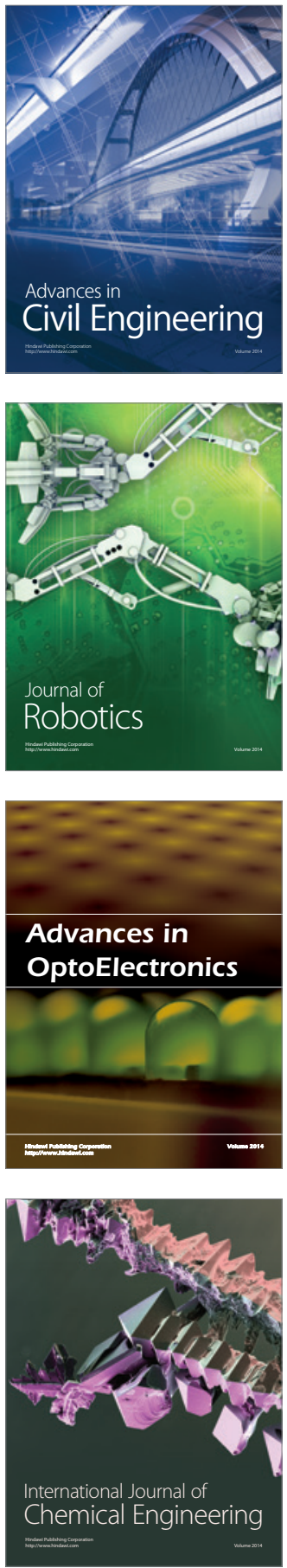

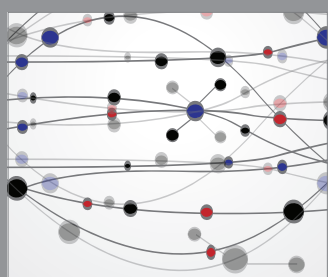

The Scientific World Journal

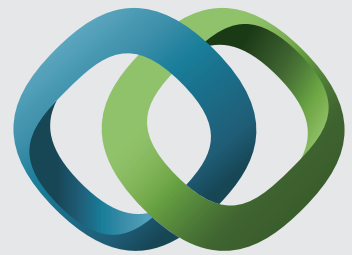

\section{Hindawi}

Submit your manuscripts at

http://www.hindawi.com
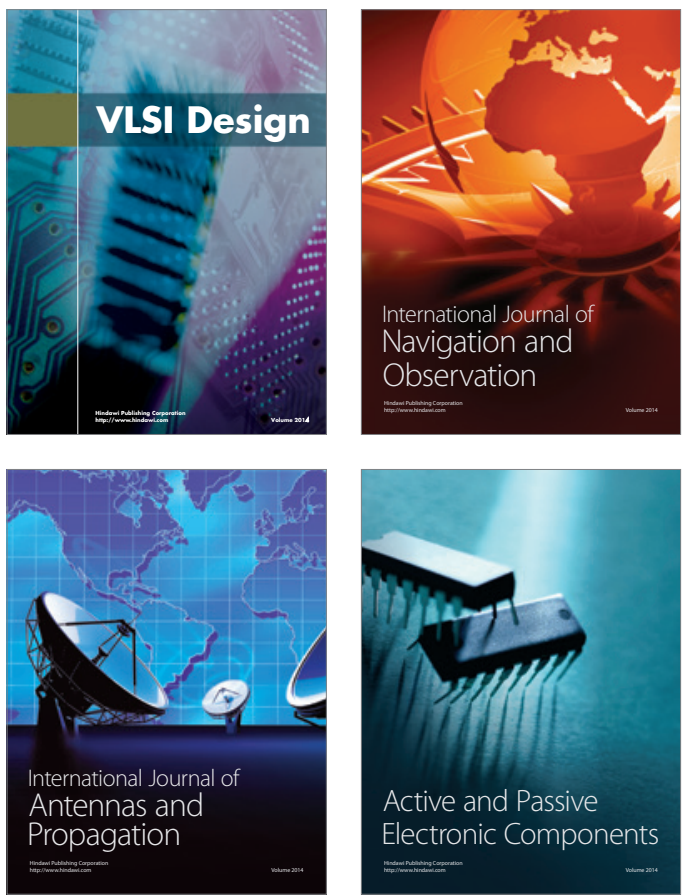
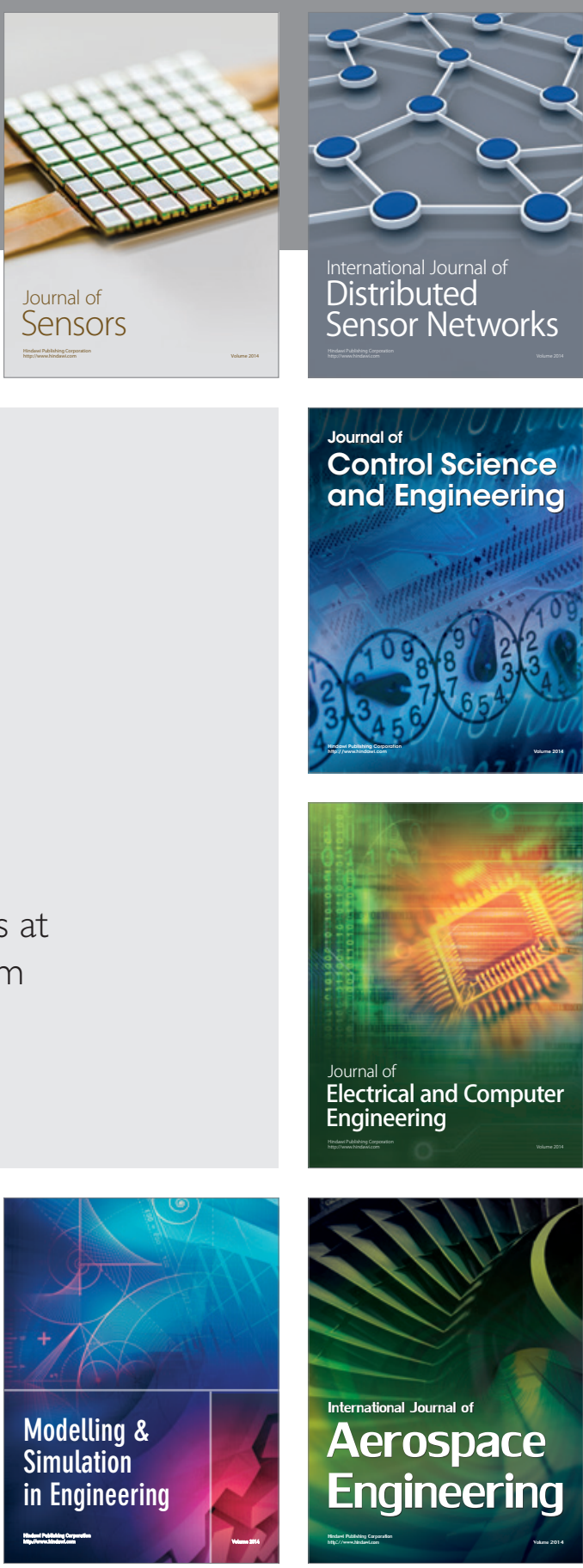

International Journal of

Distributed

Sensor Networks

Journal of

Control Science

and Engineering
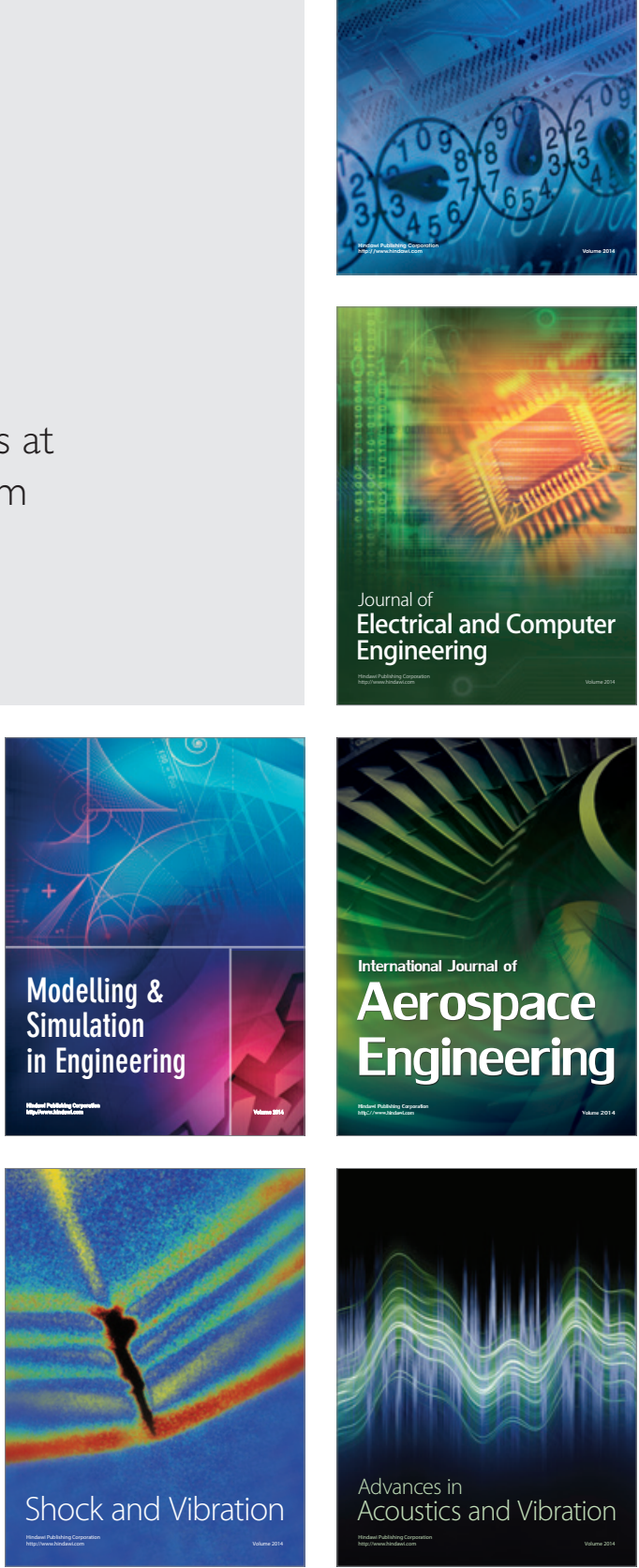